\title{
Senescence and cancer: an evolving inflammatory paradox
}

Megan Ruhland ${ }^{1}$, Lisa M. Coussens ${ }^{2, \#}$, Sheila Stewart ${ }^{1,3, \#}$

1Department of Cell Biology and Physiology, Washington University School of Medicine, Saint Louis, MO

2Department of Cell, Developmental \& Cancer Biology, and Knight Cancer Institute, Oregon Health \& Science University, Portland, OR

${ }^{3}$ Departments of Cell Biology and Physiology, and Medicine, ICCE Institute, Washington University School of Medicine, Saint Louis, MO

\#Address for correspondence:

Lisa M. Coussens, Ph.D.

Cell, Developmental \& Cancer Biology

Knight Cancer Institute

Oregon Health \& Science University

3181 SW Sam Jackson Park Road

Portland, OR 97239-3098

Voice: $503-494-7811$

Fax: 503-494-4253

email: coussenl@ohsu.edu

Sheila A. Stewart, Ph D.

Washington University

BJC-IH ICCE Institute

660 S. Euclid Ave., Box 8228

St. Louis, MO 63110-1093

Voice: $314-362-7437$

Fax: 314-362-0152

Email: sheila.stewart@wustl.edu 


\section{ABSTRACT}

The senescent phenotype was first describe in 1961 as a phenomenon characterized by the cessation of cellular division. After years of debate as to whether it represented a tissue culture artifact or an important biological process, it is now appreciated that senescence plays an important role in tumorigenesis. Further, senescence is integral to normal biological processes such as embryogenesis and the maintenance of tissue homeostasis. Now with defined roles in development, wound healing, tumor promotion and tumor suppression, it is not surprising that attention has turned to refining our understanding of the mechanisms behind, and consequences of, the induction of senescence. One emerging role for senescence lies in the ability of senescence to orchestrate an inflammatory responses: factors secreted by senescent cells have been identifed in multiple contexts to modulate various aspects of immune response. As with many of the previously described roles for senescence, the type of inflammation established by the senescence phenotype is varied and dependent on context. In this review, we discuss the current state of the field with a focus on the paradoxical outcomes of the senescence-induced inflammatory responses in the context of cancer. A more complete understanding of senescence and an appreciation for its complexities will be important for eventual development of senescence-targeted therapies.

\section{The Senescence Phenotype}


Cellular senescence has documented roles in halting tumor development through its induction of an irreversible cell cycle arrest and ability to elicit potent immune- mediated clearance of incipient tumor cells (1-6); however, like many cellular processes, the complexities that surround the senescence phenotype are still not fully understood. While there is convincing evidence that senescence can function in a cell-autonomous manner to halt tumor development in preneoplastic epithelial cells, the nuanced outcomes of cellular senescence both in the stromal compartment and the epithelial compartment have proven varied, and at times paradoxical (7-9). Indeed, in xenograft settings stromal senescence can be potently tumor promoting. More recently, senescent cells have been found to modulate host immune responses, in addition to roles in regulating tumor cell intrinsic mechanisms fostering neoplastic progression (3, 4, 9-15). Interestingly, there is now evidence that this interplay between senescent cells and immune cells is bidirectional given that tumor-infiltrating Gr-1+ myeloid cells can antagonize senescence induction within tumor epithelium (16). This review focuses on the impact senescent cells have on host immune responses, and how this relationship impacts overall tumorigenesis.

\section{Hallmarks of the senescence phenotype}

There are many inducers of the senescence phenotype: oncogene-induced senescence (OIS), telomere dysfunction (known as replicative senescence, (RS)), therapy-induced senescence (TIS), and chronic stress stimuli such as high levels of reactive oxygen species (ROS), among others $(1,2,17-21)$. All of these inducers converge upon activation of a chronic DNA-damage response (DDR), induction of the tumor suppressor 
$p 53$, or independent activation of the cell cycle inhibitor p16 INK4A $(p 16)$. Activation of p16 drives irreversible exit from the cell cycle through inhibition of CDK4- and CDK8mediated phosphorylation of the retinoblastoma protein $(R b)(11,22,23)$.

While no single marker is sufficient to identify senescent cells, a combination of biomarkers and cellular characteristics identify senescent cells both in vivo and in vitro. Importantly, these markers are sufficient to identify senescent cells regardless of the senescence inducer or cell type. Typically, senescent cells are immunoreactive for senescence-associated beta-galactosidase (SA- $\beta$ gal), express $p 16$, display a flattened and enlarged morphology in vitro, and express an altered gene expression profile, e.g., the senescence-associated secretory phenotype (SASP), activated in response to chronic DNA-damage response (DDR), and sustained by activation of the stress kinase p38MAPK (p38) (24-28). Adding further complexity to understanding senescence is the fact that SASP itself varies depending on the cell type being examined (29-37). Of note, a recent study reported that the SASP of oncogene-induced senescent cells propagates the senescence phenotype to neighboring cells via paracrine mechanisms highlighting a particular importance for interleukin (IL)-1 $1 \alpha$ in this process (38). In any case, it is the SASP that functionally dictates the striking diversity in cell-autonomous and cell-nonautonomous roles of senescence phenotypes $(26,34,35)$.

\section{Molecular mechanisms governing SASP expression}

A significant body of literature exists concerning the molecular control of the SASP (26, 28, 32, 39-41). Though many questions still remain, it is known that robust SASP 
expression results from chronic activation of the DDR, in response to DNA damaging agents, expression of oncogenes or loss of tumor suppressors, increased ROS, telomere dysfunction, and other prolonged cellular stresses $(26,31,32,34,39,42)$. In most settings, this requires activation of ataxia-telangiectasia mutated (ATM) and subsequent activation of nuclear factor kappa B signaling (NFkB) $(41,43)$. Once established, sustained activation of p38 plays a pivotal role in maintaining expression of a significant number of SASP factors though not all SASP factors require activation of NFKB or p38 $(26,41,43)$. Interestingly, and often confusing to many, p53 plays a key role in suppressing the SASP; indeed, loss of p53 in the face of a chronic DDR leads to increased expression of a cadre of SASP factors $(26,32)$.

\section{Multifaceted Roles for Senescence}

While many studies focus on roles for senescence in tumor promotion and progression, senescence was first discovered as a potent tumor suppressive mechanism $(3,4,44)$. Senescence within preneoplastic cells limits their expansion and consequently is important for maintenance of tissue integrity $(7,8)$; senescence, in this way, poses a barrier to incipient tumor development. Indeed, it is necessary for neoplastic cells to bypass the senescence phenotype, typically through loss of p53 and/or Rb, for preneoplasia to progress to frank neoplasia, and on to malignancy $(44,45)$. In addition to limiting replicative capacity, activation of senescence within certain neoplastic cell types, under specific contexts, leads to increased expression of inflammatory cytokines that elicit immune-mediated tumor clearance $(5,6,46)$. Senescence-induced, antitumorigenic, inflammatory phenotypes will be discussed in detail below. 
Developmentally programmed senescence

There are several roles emerging for senescence outside the context of tumor development; a new role for senescence was recently discovered in embryologic development. Two seminal papers reported that developmentally programmed senescence occurs during embryogenesis at multiple sites and is important for patterning of mammalian embryos $(47,48)$. This senescence program is dependent on p21 but differs from oncogene-induced senescence (OIS) or replicative senescence (RS) in that it is independent on DDR signaling and p53. In development, these senescent cells express a SASP with many overlapping factors with OIS SASP. Storer and colleagues, in conjunction with the findings from Muñoz-Espín and colleagues, revealed that senescence induction during development elicits macrophage-mediated senescent cell clearance where clearance of senescent cells results in tissue remodeling necessary for normal morphogenesis $(47,48)$. These findings support the notion that senescence activation in post-embryonic tissues marks an evolutionarily coopted mechanism of an otherwise necessary developmental process. It however remains unclear how SASP mechanistically results in recruitment of macrophages during embryogenesis. It is possible that studies focused on senescent cell clearance from tumors will provide insights into this developmental process.

\section{Senescence promotes wound repair}

In addition to development, senescence induction was recently discovered to play an integral part in the maintenance of tissue homeostasis where Demaria and colleagues 
(49) reported an essential role for senescence in optimal wound repair. Using a model of cutaneous injury, these investigators reported that following wounding, rapid induction of senescence was detected in both endothelial cells and fibroblasts. In this system, senescence induction accelerated the rate of healing by SASP factor, plateletderived growth factor AA (PDGF-AA), mediated myofibroblast differentiation. Wounds depleted or devoid of senescent cells exhibited delayed kinetics in wound closure, thus identifying a unique and non-deleterious role for the SASP in adult tissues (49). Whether wound-induced senescence modulates the host immune response remains an interesting and important question.

\section{Dual roles for senescence in tumorigenesis}

New evidence indicates beneficial outcomes for the senescence program in addition to its well-accepted, and first noted, role in cell-autonomous inhibition of neoplastic cell proliferation $(3,4,44)$. However, research from several groups over the past two decades has convincingly demonstrated a paradoxical role for senescence emanating from its distinct secretory profile $(9,26,34,35)$. A clear, cell-non-autonomous, role exists for senescence in neoplastic progression of solid tumors $(26,50-52)$. SASP factors such as osteopontin (OPN), amphiregulin (AREG) and GROa can drive proliferation of preneoplastic and neoplastic cells $(33,42,53)$. Extracellular matrix modifying enzymes, such as the canonical SASP factor matrix metalloproteinase (MMP)-3, disrupts normal tissue structure and drives invasive properties along with factors such as IL-6 and IL-8 $(26,54,55)$. Growth of tumors is further supported through increased angiogenesis derived from heightened expression of SASP factor 
vascular endothelial growth factor (VEGF) (56). Evidence from in vitro studies indicates that IL-6 and IL-8 can also promote epithelial-to-mesenchymal transition (EMT) from premalignant cells and non-aggressive tumor epithelial cells (26). In this way, senescent stromal cells were found to function analogously to cancer-associated fibroblasts (CAFs), which also express a plethora of tumor promoting factors $(10,28$, $33,57,58)$.

While the tumor-promoting functions of the SASP are varied, the majority of the research elucidating these phenotypes relied on xenograft models and immunecompromised mice $(42,50,51,53)$; thus, in recent years, introduction of immunecompetent murine models has revealed additional roles for senescence in the inflammatory response associated with cancer $(5,6,30,47-49)$. It had long been hypothesized that senescent cells could impact tumor-associated inflammation given that the SASP is enriched in many cytokines and chemokines. However, it was not until recently that the complexities of senescence-orchestrated inflammation have been brought to light. As is the story of senescence in nearly all contexts investigated, senescence-associated inflammation exerts its effects in a context-dependent manner

resulting in paradoxical outcomes dependent on the cells and tissue of origin $(5,6,30$, 47-49).

\section{A Role for Senescence in Inflammation: The SASP}

Inflammation, like senescence, within the context of cancer is a double-edged sword (reviewed in $(59,60))$. Indeed there are clear roles for acute inflammation in the 
detection of early preneoplastic lesions where immune surveillance and lymphocytemediated tumor cell clearance are an indispensible part of anti-tumor immunity (61). However, many studies have reported that chronic inflammation can be a key driver of tumor development and indeed a hallmark characteristic of cancer (62-66). In addition, the microenvironment of many established tumors is often immunosuppressive with subsets of myeloid, $T$ and $B$ cell lineages actively limiting cytotoxic $T$ cell function and subsequent tumor elimination $(67,68)$; thus, understanding the cause and context of different types of inflammation will be integral for development of successful, immunetargeted cancer therapies. The senescence-induced inflammatory response and its dichotomistic consequences for tumorigenesis will be the focus of the remainder of this review.

Understanding senescence-driven inflammation, in the context of tumor development, requires teasing apart the types of inflammation elicited by senescent cells. Initial studies have indicated a less than clear-cut and singular definition of senescence-driven inflammation. As highlighted in detail in the examples that follow, it may be possible that the dichotomy of outcome (tumor promotion or tumor regression) is also dictated by the tissue of origin, the cell of origin, the penetrance of senescence (i.e. number of senescent cells present in the tissue), the senescence-inducer, or an even broader influence such as systemic differences in the host ranging from allele differences, epigenetic differences and even differences emanating from the microbiome.

\section{Senescence in Context}




\section{Senescence-inflammatory response in epithelium}

Looking exclusively at senescence-driven inflammation developing in response to senescent epithelial cells, we are presented with two distinct and contradictory outcomes. Several studies have reported clearance of senescent neoplastic epithelial cells and malignant tumor cells that represent potent barriers for ongoing tumor development $(6,46)$. One of the first studies to demonstrate this phenomenon was performed by Xue and colleagues (6) utilizing a "tet-off" RNAi system allowing inducible silencing of $\mathrm{p} 53$. These authors demonstrated that reactivation of $\mathrm{p} 53$ in $\mathrm{H}-\mathrm{Ras}^{\mathrm{V} 12}$ expressing tumorigenic hepatoblasts, following intrasplenic injection and seeding in liver, led to robust senescence and tumor regression. Tumor regression was elicited through the SASP-mediated recruitment of innate immune cells and subsequent clearance of the tumor. Interestingly, this study was performed in athymic, nu/nu mice, thus indicating that presence of adaptive immunity was not required for clearance of tumor cells. Using the same Ras-driven, p53-tet-off system, a recent follow up study revealed that natural killer (NK) cells are the innate immune cell mediating rapid elimination of senescent tumor cells (Fig. 1A) (46); in the absence of NK cells, tumors still regressed but with slower dynamics, where neutrophil and/or macrophage activities were also implicated in clearance. Using a subcutaneous tumor model in RAG2deficient mice (lacking $B$ and $T$ lymphocytes), it was subsequently reported that subcutaneous tumors retained a similar innate immune profile as observed in liver, and that in that system, the SASP factor chemokine CCL2 was responsible for NK cellmediated, senescent tumor cell clearance (Fig. 1A) (46). How an adaptive immune response might have impacted this response however, remains an open question. 
In a separate study also utilizing OIS in liver epithelium via intrahepatic delivery of virally expressed oncogenic Nras ${ }^{G 12 V}$, as opposed to implantation of malignant tumor cells, senescence was found to play a key role in limiting the onset of tumor development. In this study, premalignant, senescent hepatocytes provoked what the authors' termed "senescence surveillance" (5). This surveillance mechanism resulted from SASPmediated, antigen specific, CD4 ${ }^{+} \mathrm{T}$ helper type $1\left(\mathrm{~T}_{\mathrm{H}} 1\right)$ cell activation, however $\mathrm{CD}^{+} \mathrm{T}$ cells required the presence of monocytes/macrophages to induce clearance of senescent premalignant cells, thus resulting in inhibition of tumor formation (Fig. 1B). And while the previous study (46) reported senescent cell clearance dependent solely on innate immunity, this later study indicated that not only were both arms of the immune system required for senescence surveillance, but also that antigen specificity played a role (5). Mechanisms underlying why adaptive immunity exerts a role in one system and not the other remains an outstanding question, but one possibility may lie in the penetrance of senescence activation within the tissue. Kang et al. demonstrated mosaic activation within the liver, while Xue et al. utilized a model system inducing over $80 \%$ SA- $\beta$ gal positivity throughout the tissue $(5,6)$. In addition, these inconsistencies may result from differences in time of onset of senescence (premalignant vs. malignant). Regardless, together these data indicate a beneficial role for senescence in restriction of hepatocellular carcinoma $(\mathrm{HCC})$ development that is, in part, reliant on immune-mediated mechanisms. 
Moving to studies using models outside of HCC, a different outcome for the senescence-inflammatory response emerges. In a prostate cancer model, PTEN-loss induced cellular senescence (PICS) revealed that PTEN-null tumors demonstrated a high degree of senescence induction within the initiated epithelium. In this model, the senescent tumor cells, through activation of Jak2/Stat3 signaling, established immunosuppressive inflammation in tumor microenvironments that promoted tumor progression and enhanced chemoresistance to docetaxel therapy (Fig. 1C). Intriguingly, this pro-tumorigenic inflammatory response could be reversed using pharmacological inhibition of JAK2, raising the possibility that the senescence phenotype may represent a novel therapeutic target (30). This study indicated that senescent tumor cells may provide protection for non-senescent tumor cells via inhibition of immune destruction thus leading to risk of tumor recurrence.

The SASP has also been found to protect tumor cells from chemotherapy, and not surprisingly, depending on the tumor model used, provides insight into two distinct mechanisms of chemoprotection. Using a mammary adenocarcinoma tumor model, Jackson et al. suggested that SASP factors derived from chemotherapy-induced, senescent malignant mammary epithelial cells directly promote non-senescent tumor cell proliferation and thus relapse following chemotherapy (69). Toso and colleagues reported that, in the context of PICS, SASP can also drive development of an immunosuppressed microenvironment; following chemotherapy, SASP-induced inflammation inhibited the anti-tumor immune response thus limiting detection and clearance of remaining, non-senescent tumor cells $(30,69)$. Another interesting finding 
from this study was evidence that the mode of senescence activation could vary the senescence-inflammatory response. Indeed, the findings using PICS in prostate epithelium were not corroborated using OIS. PbKras ${ }^{\mathrm{G} 12 \mathrm{~V}}$-driven, senescent prostate tumors lacked robust induction of Stat3 that mediated the SASP expression profile necessary to establish the immunosuppressive immune infiltration in PICS-driven senescence (30). These results highlight the important role the senescence inducer plays in the inflammatory outcome and tumor progression, and thus adds an additional layer of complexity to the impact of senescence on the host immune response.

Distinctly different from the studies above in which used liver and prostate models, Pribluda and colleagues defined a unique type of inflammation in colorectal cancer induced by the senescence-inflammatory response (29). What these authors termed the senescence-associated inflammatory response (SIR), represents a secretory profile elicited from stressed epithelium but, through loss of p53, can be uncoupled from growth arrest induced by the canonical senescence phenotype, and thus can result in accelerated tumor growth and invasiveness (Fig. 1D). Interestingly, this may be similar to the uncoupling of the senescent growth arrest and induction of SASP noted earlier by Campisi and colleagues who reported that p53 plays an important role in restraining the SASP $(26,32)$. In the Pribluda study, the authors focused on direct tumor promoting effects of the SIR rather than involvement of inflammatory infiltrates - it would be interesting to explore whether the SIR has any pronounced role in immune cell recruitment or function. In addition, the direct tumor promoting, para-inflammation 
observed in this study, was reversed through treatment with anti-inflammatory drugs providing a therapeutic opportunity (Fig. 1D) (29).

Senescence-inflammatory response in tumor stroma

Epithelial compartment-focused studies have revealed variations in inflammatory responses and tumor progression dependent on tissue type, senescence-inducer and time of senescence induction (pre-malignant vs. malignant). From these collective studies, it is not unexpected that a shift in focus from senescence in epithelium to senescence in stroma would serve to further complicate the definition of the senescence-inflammatory response. Indeed, recent studies have highlighted the myriad of effects that senescence exerts on stromal-induced inflammation. Initial studies have been restricted to the liver microenvironment with hepatic stellate cells (HSCs) the focus, but even in this single tissue, the inflammatory response is multidimensional $(70$, 71).

Recently, Lujambio et al. delineated an inflammatory mechanism in liver resulting from non-cell-autonomous functions of p53. This study demonstrated that p53-dependent senescence induction in HSCs resulted in the enrichment of M1-polarized macrophages within liver (70) (for a review of macrophage polarization, please see (72)). Using conditioned-medium (CM) from senescent HSCs or proliferating, non-senescent, HSCs, SASP factors derived from senescent HSCs induced M1-polarized bone marrowderived macrophages that had increased capacity to eliminate senescent HSCs in vitro as compared to non-senescent HSCs CM, which polarized macrophages towards an 
M2 phenotype. Addition of premalignant hepatocytes to macrophage cultures revealed that senescent HSC-CM-mediated M1 enrichment led to a reduction in the proproliferative environment observed when non-senescent, HSC-CM and M2 macrophages were instead present. Interestingly, the M1 polarization preference observed under senescent conditions also occurred in vivo and correlated with decreased diethylnitrosamine (DEN)-induced HCC (70). Thus, this study concluded that the appearance of senescent HSCs altered the immune microenvironment by favoring the presence M1-polarized macrophages that effectively limited proliferation of initiated hepatocytes, and thereby limited development of HCC (Fig. 2A). M1 macrophages in this scenario were also likely to be less immune ( $T$ cell) suppressive (68), although this aspect of macrophage functionality was not specifically addressed.

In a DMBA (7,12-dimethylbenz(a)anthracene)-initiated model of HCC, a very different outcome was observed as compared to the above findings, where the appearance of senescent HSCs resulted in overt tumorigenesis (71). In this setting, DMBA leads to oncogenic Ras mutations thus posing a different underlying mutational profile than that found following DEN treatment, which initiates tumors following DNA-adduct formation and spontaneous mutations $(71,73)$. In addition to differences in tumor initiator, the DMBA study focused on the liver microenvironment of obese mice, thus complicating the model. In this study, DMBA treatment resulted in HCC in obese mice, but the same treatment failed to induce $\mathrm{HCC}$ in control mice, indicating that the liver microenvironment is significantly different in obese versus non-obese mice - elucidation of the mechanism underlying obesity-induced HCCs revealed that obese livers 
accumulated senescent HSCs via gut microbiotic-driven changes in enterohepatic circulation of deoxycholic acid (DCA). DCA is known to induce DNA-damage and thus chronic DDR-activation was proposed as the mechanism of senescence induction (71). These important findings have thus provided evidence supporting a role for the microbiome in outcomes of senescent stromal-associated inflammation. Senescenceinduction in this context was dependent on DCA, which induced HSC senescence. SASP expression was required for HCC given that inhibition of SASP abrogated tumor development (Fig. 2B) $(71,74)$. Differences in the DMBA and DEN models indicate that the mutational profile of the responding, initiated, epithelium along with systemic differences in the host, including microbiotic composition, may contribute to distinct phenotypes of stromal senescence-derived inflammatory responses. The specific influence on the host immune response using this model system is likely to be critical, but has yet to be investigated.

\section{Age-related inflammation and cancer}

Interestingly, the natural aging process has often been described as a condition of chronic inflammation with systemic levels of factors such as IL-6 and tumor necrosis factor (TNF)a rising in serum as a consequence of age (74-76). In addition to serum level cytokine increases, elevated numbers of circulating myeloid cells with $\mathrm{T}$ cell suppressive features have also been detected (77). Age-related inflammation is linked to a number of degenerative diseases associated with aging including Alzheimer's and Parkinson's disease $(78,79)$. In these particular diseases, it is unclear whether inflammation existed prior to disease onset or is rather a confounding result of the 
disease itself. The potential contribution of the immune system towards development of these diseases will be an important area for future research.

Much more is known regarding the link between chronic inflammation and cancer; chronic inflammation is now appreciated for being associated with the onset and progression of virtually all solid tumors $(66,80)$. Ulcerative colitis and Crohn's disease greatly increase the risk of colon cancer (81), whereas liver carcinoma incidence is increased by Hepatitis $C$ infection (82), and the leading cause of gastric cancer has been traced to chronic infection by Helicobacter pylori (83). Unlike the chronic inflammation associated with a defined inducer such as an autoimmune condition or infection by pathogen, the role of age-associated, chronic inflammation, in cancer incidence is poorly understood.

Senescent cells may provide the link between age-related increases in inflammation and cancer. Indeed, senescent cells accumulate with age in multiple tissues including skin, breast, liver and lung (24, 84-86). This accumulation of senescent cells along with their robust expression of inflammatory mediators as part of the SASP, implicates senescence as a player in age-related chronic inflammation. While it is clear from the studies discussed, that senescent cells are capable of modulating inflammation, what is unclear is the type of inflammation that would be expected to be associated with agerelated senescence, where senescent cells associated with aging are likely accumulating due to a combination of inducers. DNA-damage, telomere dysfunction, chronic exposure to stressors such as ROS could all be contributing to increases in 
senescence both in stroma, as well as epithelium. As illustrated in the examples above, the inducer of senescence as well as the target cell can both produce a varied SASP expression profile and thus differentially impact the host immune response $(5,30,71)$. How the combination of these assorted senescence inducers impacts immune recruitment, function and subsequent tumorigenesis in an aging tissue is likely to be heterogeneous, and thus the outcome mixed and partially dependent on the compilation of cell autonomous mutations present in initiated cells (Fig. 3). Moreover, it is likely that age-related immune dysfunction is also involved given that the rate of senescence accumulation may be in part a reflection of reduced clearance via a weakened immune response.

Considering that the mutational profile of responding initiated cells plays a role in senescence-driven inflammation, it is conceivable that age-associated senescence will dictate different inflammatory outcomes based on cell type, tissue type and tumor type. It is clear that more investigation is needed to fully appreciate the role of inflammation in the age-associated accumulation of senescence, in both the stromal and epithelial compartments. That said, initial studies have shown promise for senescence- (namely SASP) targeted therapies for improved tumor outcome. Given recent evidence revealing elimination of $\mathrm{p}^{16^{+}}$cells from tissues in mice results in reversal of several agerelated pathologies (87), it is possible that in humans, therapies targeting the deleterious functions of the SASP may prove beneficial not only for cancer, but for other diseases as well. 


\section{Opportunities and Obstacles for therapeutics}

The documented pro- and anti-tumorigenic functions of SASP-induced inflammation make therapeutic development challenging, and indicate that optimal SASP "reprogramming" may result in a tailored secretory profile allowing for senescence-induced anti-tumorigenic benefits, while limiting tumor-promoting inflammation. Senescencetargeted therapies may be particularly potent when paired with chemotherapy and/or radiation therapy in the context of cancer. In this case, therapy-induced senescence (TIS) has proven to be beneficial in its initial characterization, but presents a risk over time $(30,69,88,89)$. Using an E $\mu$-Myc murine model of lymphoma, exogenous B cell lymphoma 2 (Bcl2) protein expression resulted in improved outcome following therapy

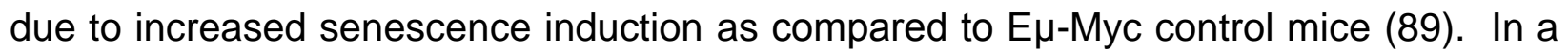
human system where the conditions surrounding TIS may or may not result in clearance, unresolved inflammation and pro-tumorigenic SASP secretion presents a risk. Others have reported evidence for chemo-protection via SASP-factors $(30,69$, 90); SASP-mediated protection and even promotion of escaped malignant cells could result in aggressive relapse. Indeed, one of the most robust ways to induce a potent SASP is through use of chemotherapeutic agents. Indeed, treatment with bleomycin, as well as docetaxel, induces a potent SASP that stimulates tumor growth in xenograft models (bleomycin), and in vivo using an MMTV-Wnt1 mammary tumor model (docetaxel) $(28,69)$.

In addition, age-associated senescence accumulation poses an interesting target for preventative therapies. It has been suggested by others that enhanced induction of 
senescence within some tumor types may be beneficial for outcomes via solicitation of anti-tumor inflammation and clearance $(70,88)$. However, given the many protumorigenic functions of unresolved senescence-induced inflammation, such a therapeutic approach may present significant obstacles.

The SASP has a multitude of factors that have been characterized as driving tumorpromoting inflammation. IL-6 is an important mediator of the SASP's tumor promoting phenotype $(34,35,91)$; this is an attractive target for inhibition using monoclonal antibody therapy. Siltuximab, an anti-IL-6 antibody, and tocilizumab, an anti-IL-6 receptor antibody, are both currently approved for treatment of other pathologies (92, 93). Upstream of IL-6, IL-1 $\alpha$ could also be targeted - as is the case with IL-6, an IL-1 $\alpha$ receptor monoclonal antibody is already used clinically (94). The use of SASP-factor specific therapies for senescence would require careful consideration of the tissue type and cell of origin in order to achieve maximal benefit.

Other targets outside of cytokines exist that may provide broad inhibition of inflammatory SASP factors. p38MAPK (p38) inhibition was found to limit SASP factor mRNA stability critical for maintenance of NFKB-dependent cytokine and chemokine upregulation. Treatment of senescent stromal cells with p38-inhibitors led to amelioration of tumor promoting effects of the SASP in vitro, as well as in a xenograft system (28). In addition, a recent study reported that mTOR is a potent target that provides inhibition of the pro-tumorigenic functions of the SASP where SASP inhibition resulted from suppression of IL-1 $\alpha$ translation and a subsequent decrease in NFKB- 
dependent cytokine secretion. Interestingly, the effects of rapamycin were notably durable with suppression of the SASP maintained at approximately $80 \%$ for up to 7 days post-treatment and taking 3 weeks to return to levels observed in untreated cells (95). Given the types of factors with reduced expression, it is likely that in an immunecompetent setting, inflammation would also be impacted. Whether such approaches would have detrimental effects on important functions such as wound healing will be an important question to address.

In cases where SASP establishes inflammatory microenvironments characterized by immunosuppressive cell types, such as the PICS model in prostate epithelium, there is evidence that the SASP can be reprogrammed to stimulate tumor cell rejection. In this model, treatment with the JAK2 inhibitor NVP-BSK805 (JAK2i) resulted in a reversal of the immunosuppressive phenotype and synergized with chemotherapy to produce more favorable tumor outcome. Following treatment with the JAK2i, tumors displayed robust infiltration by $T$ cells and elevated granzyme B staining, correlating with significantly decreased tumor burden (30). This type of reprogramming poses an interesting possibility - that stimulating the "right" type of inflammation may be the best of both worlds to enable clearance of tumor cells, and at the same time lift suppression driven by subsets of recruited myeloid cells. While depletion of suppressive myeloid cells was noted in the JAK2i treated tumors (30), it would be interesting to determine if these studies performed with addition of immune checkpoint blockade therapies (for a review, see (96)) that have demonstrated efficacy in multiple malignancies (97). The hope here would be that addition of immune checkpoint blockade to microenvironments with 
senescence-induced immunosuppressive cell types may benefit from reactivation of anti-tumor $\mathrm{T}$ cell responses.

Use of non-steroidal anti-inflammatory drugs (NSAIDs) has also shown promise in limiting the tumor-promoting affects of senescence in the context of colorectal cancer (CRC) (29). While NSAIDs in human studies are known to reduce risk of some cancers, and reduce recurrence of others following primary resection, the manner by which they achieve this is not entirely clear (98-102). It is possible that some of the reduced cancer risk is derived from systemic decreases in inflammation. Evidence from murine models of CRC reveled that treatment with the NSAID, sulindac resolved para-inflammation driven by the SIR, and the reduction in "smoldering" inflammation resulted in reduced tumor development (29). NSAIDs, such as aspirin and ibuprofen, are readily available compounds but more investigation is needed as to their mode of action. Moreover, adverse effects, including gastrointestinal issues result in complications for the elderly, a group of individuals that would potentially benefit from pan-inhibition of age-driven accumulating senescent cells.

\section{Conclusions}

The senescence phenotype is complicated and its impact in different tissues likely stems from variations in intrinsic SASP factor expression, as well as differences in the phenotypes of responding cells. Taken together, the studies investigating SASPmediated inflammation further highlight the complexities of the senescence phenotype. Together these results demonstrate a dynamic inflammatory response dictated by the 
cell type (stromal versus epithelial), the tissue type (liver, prostate, colon etc.), the senescence inducer (OIS, PICS, RS and others), and the mutational profile of the responding epithelium (preneoplastic versus neoplastic, DMBA-induced oncogenic Ras versus DEN-induced DNA-adducts, etc). Many questions remain as studies begin to investigate senescence-associated inflammation in contexts outside of the tumor microenvironment. This includes a provocative link to aging pathologies including ageassociated increases in cancer where senescent cells are present in stroma before any evidence of initiation from epithelium. In order to harness the potential therapeutic advantages of senescence, it is clear that a more profound understanding of the phenotype is needed both in normal tissues as well as the tumor microenvironment.

\section{ACKNOWLEDGEMENTS:}

The authors thank members of the Stewart and Coussens laboratories for critical insight and discussions, and all authors contributing to studies discussed herein, but not mentioned due to space consideration. LMC acknowledges support from the $\mathrm{NCI} / \mathrm{NIH}$, the Department of Defense Breast Cancer Research Program, the Susan G Komen Foundation, the Breast Cancer Research Foundation, a Stand Up To Cancer Lustgarten Foundation Pancreatic Cancer Convergence Dream Team Translational Research Grant (SU2C-AACR-DT14-14), the Brenden-Colson Center for Pancreatic Health and Knight Cancer Institute at OHSU. SAS acknowledges support from NIH 5 R01CA151518, an American Cancer Society Research Scholar Award, the Alvin J. Siteman Cancer Research Fund at Washington University in St. Louis, MO and Siteman Cancer Center (P30 CA091842, Eberlein, PI.) Barnes-Jewish Hospital Foundation, the 
Susan G. Komen Breast Cancer Foundation, and the Alvin J. Siteman Cancer Center at Washington University School of Medicine and Barnes-Jewish Hospital in St. Louis, MO., for stipend support to MKR.

\section{REFERENCES}

1. Di Leonardo A, Linke SP, Clarkin $\mathrm{K}$, Wahl GM. DNA damage triggers a prolonged p53-dependent G1 arrest and long-term induction of Cip1 in normal human fibroblasts. Genes \& development. 1994;8:2540-51.

2. Serrano $M$, Lin AW, McCurrach ME, Beach D, Lowe SW. Oncogenic ras provokes premature cell senescence associated with accumulation of p53 and p16INK4a. Cell. 1997;88:593-602.

3. Braig M, Schmitt CA. Oncogene-induced senescence: putting the brakes on tumor development. Cancer research. 2006;66:2881-4.

4. Campisi J. Cellular senescence as a tumor-suppressor mechanism. Trends Cell Biol. 2001;11:S27-31.

5. Kang TW, Yevsa T, Woller N, Hoenicke L, Wuestefeld T, Dauch D, et al. Senescence surveillance of pre-malignant hepatocytes limits liver cancer development. Nature. 2011;479:547-51.

6. Xue W, Zender L, Miething C, Dickins RA, Hernando E, Krizhanovsky V, et al. Senescence and tumour clearance is triggered by p53 restoration in murine liver carcinomas. Nature. 2007;445:656-60. 
7. Chen Z, Trotman LC, Shaffer D, Lin HK, Dotan ZA, Niki M, et al. Crucial role of p53-dependent cellular senescence in suppression of Pten-deficient tumorigenesis. Nature. 2005;436:725-30.

8. Braig M, Lee S, Loddenkemper C, Rudolph C, Peters AH, Schlegelberger B, et al. Oncogene-induced senescence as an initial barrier in lymphoma development. Nature. 2005;436:660-5.

9. Rodier F, Campisi J. Four faces of cellular senescence. J Cell Biol. 2011;192:547-56.

10. Alspach E, Fu Y, Stewart SA. Senescence and the pro-tumorigenic stroma. Crit Rev Oncog. 2013;18:549-58.

11. Campisi J, d'Adda di Fagagna F. Cellular senescence: when bad things happen to good cells. Nat Rev Mol Cell Biol. 2007;8:729-40.

12. Campisi J, Kim SH, Lim CS, Rubio M. Cellular senescence, cancer and aging: the telomere connection. Exp Gerontol. 2001;36:1619-37.

13. Pazolli E, Stewart SA. Senescence: the good the bad and the dysfunctional. Current opinion in genetics \& development. 2008;18:42-7.

14. Lasry A, Ben-Neriah Y. Senescence-associated inflammatory responses: aging and cancer perspectives. Trends Immunol. 2015;36:217-28.

15. Perez-Mancera PA, Young AR, Narita M. Inside and out: the activities of senescence in cancer. Nat Rev Cancer. 2014;14:547-58.

16. Di Mitri D, Toso A, Chen JJ, Sarti M, Pinton S, Jost TR, et al. Tumour-infiltrating Gr-1+ myeloid cells antagonize senescence in cancer. Nature. 2014;515:134-7. 
17. Herbig U, Jobling WA, Chen BP, Chen DJ, Sedivy JM. Telomere shortening triggers senescence of human cells through a pathway involving ATM, p53, and p21(CIP1), but not p16(INK4a). Mol Cell. 2004;14:501-13.

18. Ogryzko VV, Hirai TH, Russanova VR, Barbie DA, Howard BH. Human fibroblast commitment to a senescence-like state in response to histone deacetylase inhibitors is cell cycle dependent. Mol Cell Biol. 1996;16:5210-8.

19. Chang BD, Xuan Y, Broude EV, Zhu H, Schott B, Fang J, et al. Role of p53 and p21waf1/cip1 in senescence-like terminal proliferation arrest induced in human tumor cells by chemotherapeutic drugs. Oncogene. 1999;18:4808-18.

20. Chang BD, Broude EV, Dokmanovic M, Zhu H, Ruth A, Xuan Y, et al. A senescence-like phenotype distinguishes tumor cells that undergo terminal proliferation arrest after exposure to anticancer agents. Cancer Res. 1999;59:3761-7.

21. Qi M, Fan S, Yao G, Li Z, Zhou H, Tashiro S, et al. Pseudolaric acid B-induced autophagy contributes to senescence via enhancement of ROS generation and mitochondrial dysfunction in murine fibrosarcoma L929 cells. J Pharmacol Sci. 2013;121:200-11.

22. Kim WY, Sharpless NE. The regulation of INK4/ARF in cancer and aging. Cell. 2006;127:265-75.

23. Chicas A, Wang X, Zhang C, McCurrach M, Zhao Z, Mert O, et al. Dissecting the unique role of the retinoblastoma tumor suppressor during cellular senescence. Cancer Cell. 2010;17:376-87. 
24. Dimri GP, Lee X, Basile G, Acosta M, Scott G, Roskelley C, et al. A biomarker that identifies senescent human cells in culture and in aging skin in vivo. Proc Natl Acad Sci U S A. 1995;92:9363-7.

25. Krishnamurthy J, Torrice C, Ramsey MR, Kovalev GI, Al-Regaiey K, Su L, et al. Ink4a/Arf expression is a biomarker of aging. J Clin Invest. 2004;114:1299-307.

26. Coppe JP, Patil CK, Rodier F, Sun Y, Munoz DP, Goldstein J, et al. Senescenceassociated secretory phenotypes reveal cell-nonautonomous functions of oncogenic RAS and the p53 tumor suppressor. PLoS Biol. 2008;6:2853-68.

27. Kuilman T, Peeper DS. Senescence-messaging secretome: SMS-ing cellular stress. Nat Rev Cancer. 2009;9:81-94.

28. Alspach E, Flanagan KC, Luo X, Ruhland MK, Huang H, Pazolli E, et al. p38MAPK plays a crucial role in stromal-mediated tumorigenesis. Cancer Discov. 2014;4:716-29.

29. Pribluda A, Elyada E, Wiener Z, Hamza H, Goldstein RE, Biton M, et al. A senescence-inflammatory switch from cancer-inhibitory to cancer-promoting mechanism. Cancer Cell. 2013;24:242-56.

30. Toso A, Revandkar A, Di Mitri D, Guccini I, Proietti M, Sarti M, et al. Enhancing chemotherapy efficacy in Pten-deficient prostate tumors by activating the senescenceassociated antitumor immunity. Cell Rep. 2014;9:75-89.

31. Novakova Z, Hubackova S, Kosar M, Janderova-Rossmeislova L, Dobrovolna J, Vasicova $\mathrm{P}$, et al. Cytokine expression and signaling in drug-induced cellular senescence. Oncogene. 2010;29:273-84. 
32. Rodier F, Coppe JP, Patil CK, Hoeijmakers WA, Munoz DP, Raza SR, et al. Persistent DNA damage signalling triggers senescence-associated inflammatory cytokine secretion. Nat Cell Biol. 2009;11:973-9.

33. Bavik C, Coleman I, Dean JP, Knudsen B, Plymate S, Nelson PS. The gene expression program of prostate fibroblast senescence modulates neoplastic epithelial cell proliferation through paracrine mechanisms. Cancer research. 2006;66:794-802.

34. Acosta JC, O'Loghlen A, Banito A, Guijarro MV, Augert A, Raguz S, et al. Chemokine signaling via the CXCR2 receptor reinforces senescence. Cell. 2008;133:1006-18.

35. Kuilman T, Michaloglou C, Vredeveld LC, Douma S, van Doorn R, Desmet CJ, et al. Oncogene-induced senescence relayed by an interleukin-dependent inflammatory network. Cell. 2008;133:1019-31.

36. Hampel B, Fortschegger K, Ressler S, Chang MW, Unterluggauer H, Breitwieser A, et al. Increased expression of extracellular proteins as a hallmark of human endothelial cell in vitro senescence. Exp Gerontol. 2006;41:474-81.

37. Wajapeyee N, Serra RW, Zhu X, Mahalingam M, Green MR. Oncogenic BRAF induces senescence and apoptosis through pathways mediated by the secreted protein IGFBP7. Cell. 2008;132:363-74.

38. Acosta JC, Banito A, Wuestefeld T, Georgilis A, Janich P, Morton JP, et al. A complex secretory program orchestrated by the inflammasome controls paracrine senescence. Nat Cell Biol. 2013;15:978-90.

39. Pazolli E, Alspach E, Milczarek A, Prior J, Piwnica-Worms D, Stewart SA. Chromatin remodeling underlies the senescence-associated secretory phenotype of 
tumor stromal fibroblasts that supports cancer progression. Cancer Res. 2012;72:225161.

40. Rodier F, Munoz DP, Teachenor R, Chu V, Le O, Bhaumik D, et al. DNASCARS: distinct nuclear structures that sustain damage-induced senescence growth arrest and inflammatory cytokine secretion. J Cell Sci. 2011;124:68-81.

41. Freund A, Patil CK, Campisi J. p38MAPK is a novel DNA damage responseindependent regulator of the senescence-associated secretory phenotype. The EMBO journal. 2011;30:1536-48.

42. Coppe JP, Patil CK, Rodier F, Krtolica A, Beausejour CM, Parrinello S, et al. A human-like senescence-associated secretory phenotype is conserved in mouse cells dependent on physiological oxygen. PLoS One. 2010;5:e9188.

43. Orjalo AV, Bhaumik D, Gengler BK, Scott GK, Campisi J. Cell surface-bound IL1alpha is an upstream regulator of the senescence-associated IL-6/IL-8 cytokine network. Proc Natl Acad Sci U S A. 2009;106:17031-6.

44. Hayflick L. The Limited in Vitro Lifetime of Human Diploid Cell Strains. Exp Cell Res. 1965;37:614-36.

45. Collado M, Serrano M. Senescence in tumours: evidence from mice and humans. Nature reviews Cancer. 2010;10:51-7.

46. Iannello A, Thompson TW, Ardolino M, Lowe SW, Raulet DH. p53-dependent chemokine production by senescent tumor cells supports NKG2D-dependent tumor elimination by natural killer cells. J Exp Med. 2013;210:2057-69. 
47. Storer M, Mas A, Robert-Moreno A, Pecoraro M, Ortells MC, Di Giacomo V, et al. Senescence is a developmental mechanism that contributes to embryonic growth and patterning. Cell. 2013;155:1119-30.

48. Munoz-Espin D, Canamero M, Maraver A, Gomez-Lopez G, Contreras J, MurilloCuesta S, et al. Programmed cell senescence during mammalian embryonic development. Cell. 2013;155:1104-18.

49. Demaria M, Ohtani N, Youssef SA, Rodier F, Toussaint W, Mitchell JR, et al. An essential role for senescent cells in optimal wound healing through secretion of PDGFAA. Dev Cell. 2014;31:722-33.

50. Krtolica A, Parrinello S, Lockett S, Desprez PY, Campisi J. Senescent fibroblasts promote epithelial cell growth and tumorigenesis: a link between cancer and aging. Proc Natl Acad Sci U S A. 2001;98:12072-7.

51. Liu D, Hornsby PJ. Senescent human fibroblasts increase the early growth of xenograft tumors via matrix metalloproteinase secretion. Cancer Res. 2007;67:3117-26. 52. Bhatia B, Multani AS, Patrawala L, Chen X, Calhoun-Davis T, Zhou J, et al. Evidence that senescent human prostate epithelial cells enhance tumorigenicity: cell fusion as a potential mechanism and inhibition by p16INK4a and hTERT. International journal of cancer Journal international du cancer. 2008;122:1483-95.

53. Pazolli E, Luo X, Brehm S, Carbery K, Chung JJ, Prior JL, et al. Senescent stromal-derived osteopontin promotes preneoplastic cell growth. Cancer Res. 2009;69:1230-9. 
54. Parrinello S, Coppe JP, Krtolica A, Campisi J. Stromal-epithelial interactions in aging and cancer: senescent fibroblasts alter epithelial cell differentiation. J Cell Sci. 2005; 118:485-96.

55. Tsai KK, Chuang EY, Little JB, Yuan ZM. Cellular mechanisms for low-dose ionizing radiation-induced perturbation of the breast tissue microenvironment. Cancer Res. 2005;65:6734-44.

56. Coppe JP, Kauser K, Campisi J, Beausejour CM. Secretion of vascular endothelial growth factor by primary human fibroblasts at senescence. The Journal of biological chemistry. 2006;281:29568-74.

57. Bissell MJ, Radisky D. Putting tumours in context. Nat Rev Cancer. 2001;1:4654.

58. Tuxhorn JA, Ayala GE, Rowley DR. Reactive stroma in prostate cancer progression. J Urol. 2001;166:2472-83.

59. Schreiber RD, Old LJ, Smyth MJ. Cancer immunoediting: integrating immunity's roles in cancer suppression and promotion. Science. 2011;331:1565-70.

60. de Visser KE, Eichten A, Coussens LM. Paradoxical roles of the immune system during cancer development. Nat Rev Cancer. 2006;6:24-37.

61. Shankaran V, Ikeda H, Bruce AT, White JM, Swanson PE, Old LJ, et al. IFNgamma and lymphocytes prevent primary tumour development and shape tumour immunogenicity. Nature. 2001;410:1107-11.

62. Ernst PB, Gold BD. The disease spectrum of Helicobacter pylori: the immunopathogenesis of gastroduodenal ulcer and gastric cancer. Annu Rev Microbiol. 2000;54:615-40. 
63. Martins-Green M, Boudreau N, Bissell MJ. Inflammation is responsible for the development of wound-induced tumors in chickens infected with Rous sarcoma virus. Cancer Res. 1994;54:4334-41.

64. Farrow B, Evers BM. Inflammation and the development of pancreatic cancer. Surg Oncol. 2002;10:153-69.

65. Kuper H, Adami HO, Trichopoulos D. Infections as a major preventable cause of human cancer. J Intern Med. 2000;248:171-83.

66. Hanahan D, Coussens LM. Accessories to the crime: functions of cells recruited to the tumor microenvironment. Cancer Cell. 2012;21:309-22.

67. Rabinovich GA, Gabrilovich D, Sotomayor EM. Immunosuppressive strategies that are mediated by tumor cells. Annu Rev Immunol. 2007;25:267-96.

68. Coussens LM, Zitvogel L, Palucka AK. Neutralizing tumor-promoting chronic inflammation: a magic bullet? Science. 2013;339:286-91.

69. Jackson JG, Pant V, Li Q, Chang LL, Quintas-Cardama A, Garza D, et al. p53mediated senescence impairs the apoptotic response to chemotherapy and clinical outcome in breast cancer. Cancer Cell. 2012;21:793-806.

70. Lujambio A, Akkari L, Simon J, Grace D, Tschaharganeh DF, Bolden JE, et al. Non-cell-autonomous tumor suppression by p53. Cell. 2013;153:449-60.

71. Yoshimoto S, Loo TM, Atarashi K, Kanda H, Sato S, Oyadomari S, et al. Obesityinduced gut microbial metabolite promotes liver cancer through senescence secretome. Nature. 2013;499:97-101.

72. Sica A, Mantovani A. Macrophage plasticity and polarization: in vivo veritas. $\mathrm{J}$ Clin Invest. 2012;122:787-95. 
73. Verna L, Whysner J, Williams GM. N-nitrosodiethylamine mechanistic data and risk assessment: bioactivation, DNA-adduct formation, mutagenicity, and tumor initiation. Pharmacol Ther. 1996;71:57-81.

74. Dobbs RJ, Charlett A, Purkiss AG, Dobbs SM, Weller C, Peterson DW. Association of circulating TNF-alpha and IL-6 with ageing and parkinsonism. Acta Neurol Scand. 1999;100:34-41.

75. Paolisso G, Rizzo MR, Mazziotti G, Tagliamonte MR, Gambardella A, Rotondi M, et al. Advancing age and insulin resistance: role of plasma tumor necrosis factor-alpha. Am J Physiol. 1998;275:E294-9.

76. Wei J, Xu H, Davies JL, Hemmings GP. Increase of plasma IL-6 concentration with age in healthy subjects. Life Sci. 1992;51:1953-6.

77. Verschoor CP, Johnstone J, Millar J, Dorrington MG, Habibagahi M, Lelic A, et al. Blood CD33(+)HLA-DR(-) myeloid-derived suppressor cells are increased with age and a history of cancer. J Leukoc Biol. 2013;93:633-7.

78. Blasko I, Stampfer-Kountchev M, Robatscher P, Veerhuis R, Eikelenboom P, Grubeck-Loebenstein B. How chronic inflammation can affect the brain and support the development of Alzheimer's disease in old age: the role of microglia and astrocytes. Aging Cell. 2004;3:169-76.

79. Perry VH. Innate inflammation in Parkinson's disease. Cold Spring Harb Perspect Med. 2012;2:a009373.

80. Coussens LM, Werb Z. Inflammation and cancer. Nature. 2002;420:860-7.

81. Beaugerie L, Itzkowitz $\mathrm{SH}$. Cancers complicating inflammatory bowel disease. $\mathrm{N}$ Engl J Med. 2015;372:1441-52. 
82. Perz JF, Armstrong GL, Farrington LA, Hutin YJ, Bell BP. The contributions of hepatitis $B$ virus and hepatitis $C$ virus infections to cirrhosis and primary liver cancer worldwide. J Hepatol. 2006;45:529-38.

83. de Martel C, Ferlay J, Franceschi S, Vignat J, Bray F, Forman D, et al. Global burden of cancers attributable to infections in 2008: a review and synthetic analysis. Lancet Oncol. 2012;13:607-15.

84. Jeyapalan JC, Ferreira M, Sedivy JM, Herbig U. Accumulation of senescent cells in mitotic tissue of aging primates. Mech Ageing Dev. 2007;128:36-44.

85. Wang C, Jurk D, Maddick M, Nelson G, Martin-Ruiz C, von Zglinicki T. DNA damage response and cellular senescence in tissues of aging mice. Aging Cell. 2009;8:311-23.

86. Paradis V, Youssef N, Dargere D, Ba N, Bonvoust F, Deschatrette J, et al. Replicative senescence in normal liver, chronic hepatitis C, and hepatocellular carcinomas. Hum Pathol. 2001;32:327-32.

87. Baker DJ, Wijshake T, Tchkonia T, LeBrasseur NK, Childs BG, van de Sluis B, et al. Clearance of p16Ink4a-positive senescent cells delays ageing-associated disorders. Nature. 2011;479:232-6.

88. Ewald JA, Desotelle JA, Wilding G, Jarrard DF. Therapy-induced senescence in cancer. J Natl Cancer Inst. 2010;102:1536-46.

89. Schmitt CA, Fridman JS, Yang M, Lee S, Baranov E, Hoffman RM, et al. A senescence program controlled by $p 53$ and p16INK4a contributes to the outcome of cancer therapy. Cell. 2002;109:335-46. 
90. Gilbert LA, Hemann MT. DNA damage-mediated induction of a chemoresistant niche. Cell. 2010;143:355-66.

91. Davalos AR, Coppe JP, Campisi J, Desprez PY. Senescent cells as a source of inflammatory factors for tumor progression. Cancer Metastasis Rev. 2010;29:273-83.

92. Deisseroth A, Ko CW, Nie L, Zirkelbach JF, Zhao L, Bullock J, et al. FDA approval: siltuximab for the treatment of patients with multicentric Castleman disease. Clin Cancer Res. 2015;21:950-4.

93. Karsdal MA, Schett G, Emery P, Harari O, Byrjalsen I, Kenwright A, et al. IL-6 receptor inhibition positively modulates bone balance in rheumatoid arthritis patients with an inadequate response to anti-tumor necrosis factor therapy: biochemical marker analysis of bone metabolism in the tocilizumab RADIATE study (NCT00106522). Semin Arthritis Rheum. 2012;42:131-9.

94. Fleischmann R. Anakinra in the treatment of rheumatic disease. Expert Rev Clin Immunol. 2006;2:331-40.

95. Laberge RM, Sun Y, Orjalo AV, Patil CK, Freund A, Zhou L, et al. MTOR regulates the pro-tumorigenic senescence-associated secretory phenotype by promoting IL1A translation. Nat Cell Biol. 2015;17:1049-61.

96. Pardoll DM. The blockade of immune checkpoints in cancer immunotherapy. Nat Rev Cancer. 2012;12:252-64.

97. Ott PA, Hodi FS, Robert C. CTLA-4 and PD-1/PD-L1 blockade: new immunotherapeutic modalities with durable clinical benefit in melanoma patients. Clin Cancer Res. 2013;19:5300-9. 
98. Rothwell PM, Fowkes FG, Belch JF, Ogawa H, Warlow CP, Meade TW. Effect of daily aspirin on long-term risk of death due to cancer: analysis of individual patient data from randomised trials. Lancet. 2011;377:31-41.

99. Thun MJ, Namboodiri MM, Heath CW, Jr. Aspirin use and reduced risk of fatal colon cancer. N Engl J Med. 1991;325:1593-6.

100. Sandler RS, Halabi S, Baron JA, Budinger S, Paskett E, Keresztes R, et al. A randomized trial of aspirin to prevent colorectal adenomas in patients with previous colorectal cancer. N Engl J Med. 2003;348:883-90.

101. Fraser DM, Sullivan FM, Thompson AM, McCowan C. Aspirin use and survival after the diagnosis of breast cancer: a population-based cohort study. $\mathrm{Br} \mathrm{J}$ Cancer. $2014 ; 111: 623-7$.

102. Streicher SA, Yu H, Lu L, Kidd MS, Risch HA. Case-control study of aspirin use and risk of pancreatic cancer. Cancer Epidemiol Biomarkers Prev. 2014;23:1254-63. 


\section{FIGURE LEGENDS}

Figure 1: Senescence-inflammatory response in epithelium

A. Oncogene-induced senescence (OIS) in hepatic tumor cells, through secretion of SASP-factor CCL2, stimulates natural killer (NK) cell-mediated anti-tumor immune responses.

B. Using a model of Nras ${ }^{\mathrm{G} 12 \mathrm{~V}}$-driven, OIS, senescent premalignant hepatocytes stimulate immune surveillance by secretion of SASP factors. SASP factors results in the activation of $\mathrm{CD} 4^{+}, \mathrm{TH}_{\mathrm{H}}$ cells, which subsequently limit liver cancer development via macrophage/monocyte mediate clearance.

C. PTEN-loss induced senescent prostate tumor cells promote cancer progression by the SASP factor-mediated establishment of immunosuppressive inflammation. In this model, Jak2/Stat3 dependent SASP factor expression results in recruitment of $\mathrm{CD} 11 \mathrm{~b}+\mathrm{Gr}-1^{+}$myeloid cells which limit cytotoxic $\mathrm{T}$ cell $(\mathrm{CTL})$ function and thus tumor growth is unrestrained.

D. In a model of colorectal cancer, a SASP related secretory profile, termed the senescence-associated inflammatory response (SIR), is secreted by stressed epithelial cells. The SIR promotes tumorigenesis following the loss of p53 expression. Treatment with nonsteroidal anti-inflammatory drugs (NSAIDs) can reverse the tumor promoting effects of the SIR. 


\section{Figure 2: Senescence-inflammatory response in tumor stroma}

A. In a diethylnitrosamine (DEN)-induced hepatocellular carcinoma (HCC) model, p53-dependent, senescent hepatic stellate cells promote the enrichment of M1polarized macrophages. M1 macrophages limit the proliferation of initiated epithelial cells and consequently restrict HCC development.

B. A DMBA (7,12-dimethylbenz(a)anthracene)-initiated model of HCC demonstrated that livers of obese mice were predisposed to tumor development via DCA (deoxycholic acid)-induced senescent hepatic stellate cells. DCA was produced by the gut microbiota and resulted in increased senescence in the liver. The senescent stellate cells promoted tumorigenesis by the secretion of SASP factors.

\section{Figure 3: A role for senescence in age-related tumor development}

Senescent cells accumulate in tissues with age but little is known regarding the impact of this phenomenon on inflammation and cancer development. SASP factors can both promote and restrict tumor development through modulation of immune cells. The types of inflammation elicited by senescence in aging microenvironments as well as the consequence of this inflammation on tumorigenesis remain open questions. 
rIGUKE I

A. Senescence-mediated tumor suppression in the liver epithelium

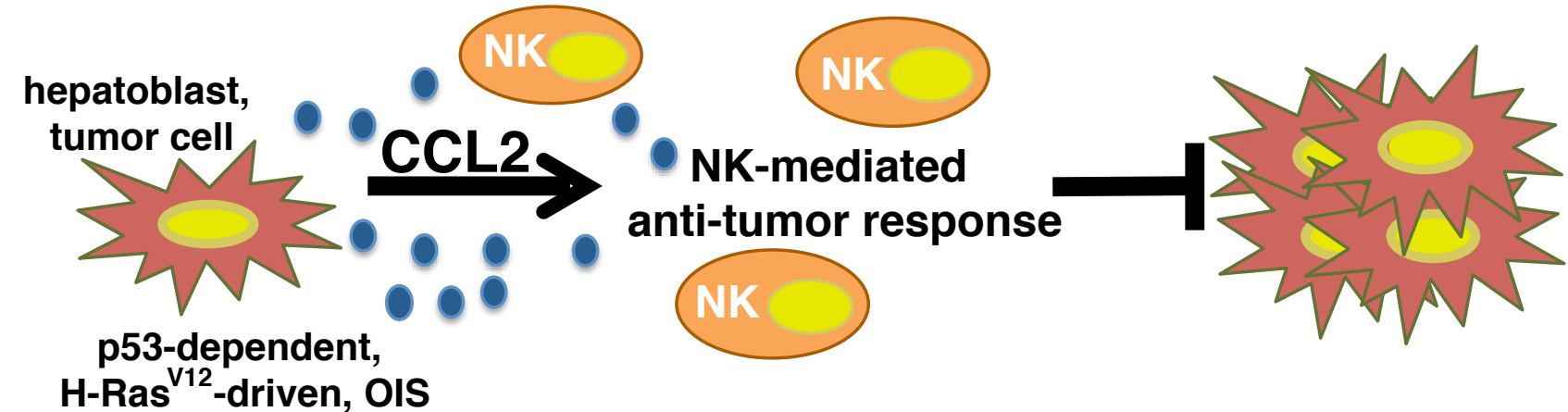

B. Senescence surveillance restricts tumor growth in liver epithelium

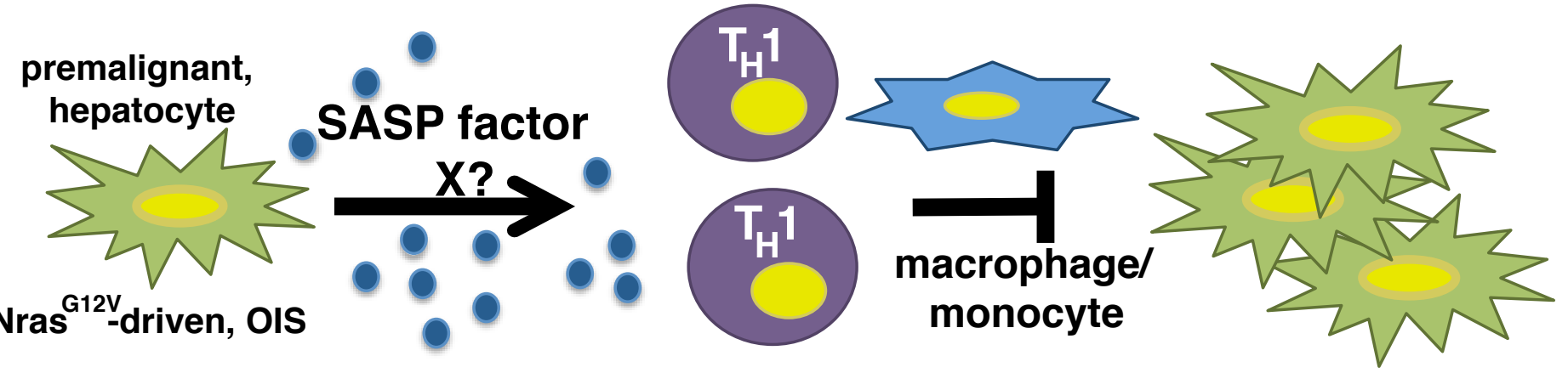

C. Senescence-mediated tumor promotion in prostate epithelium

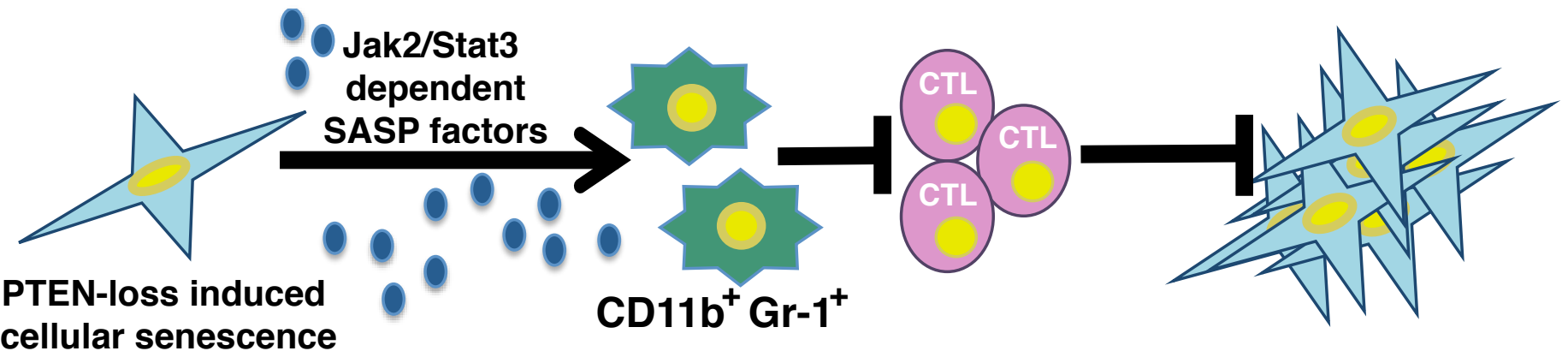

D. Senescence-mediated tumor promotion in colorectal cancer
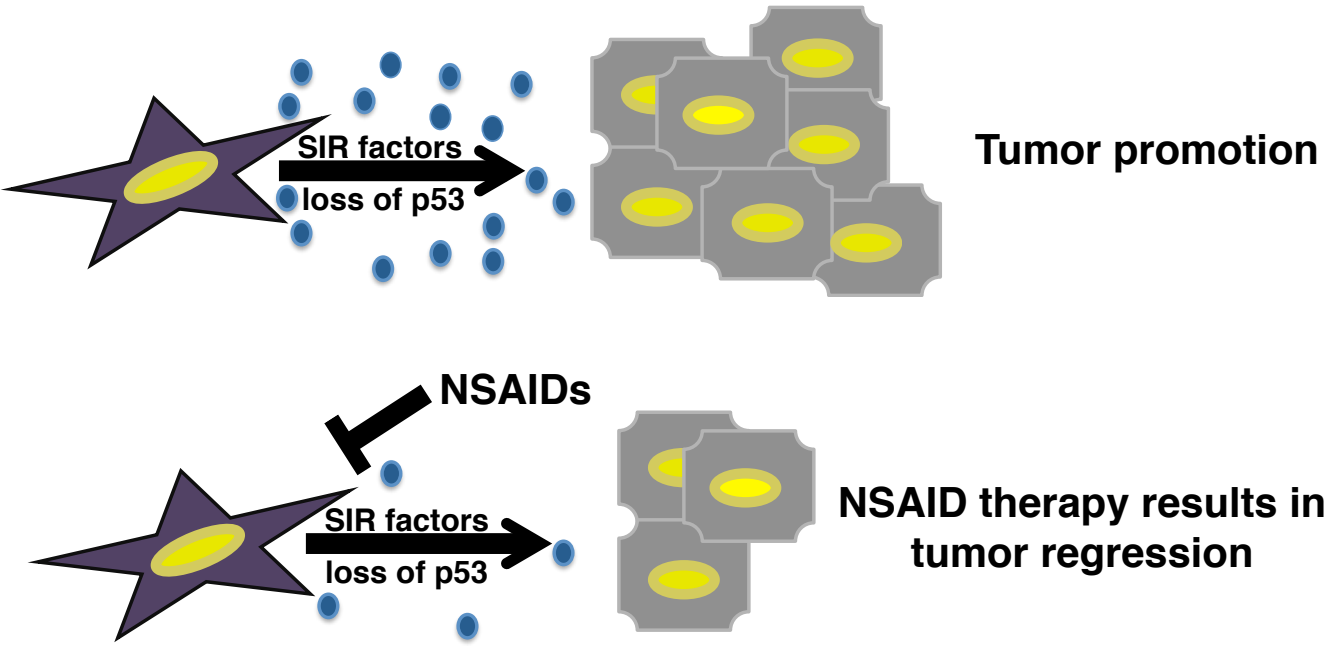

NSAID therapy results in tumor regression 


\section{Figure 2}

A. Senescent hepatic stellate cells reduce incidence of HCC

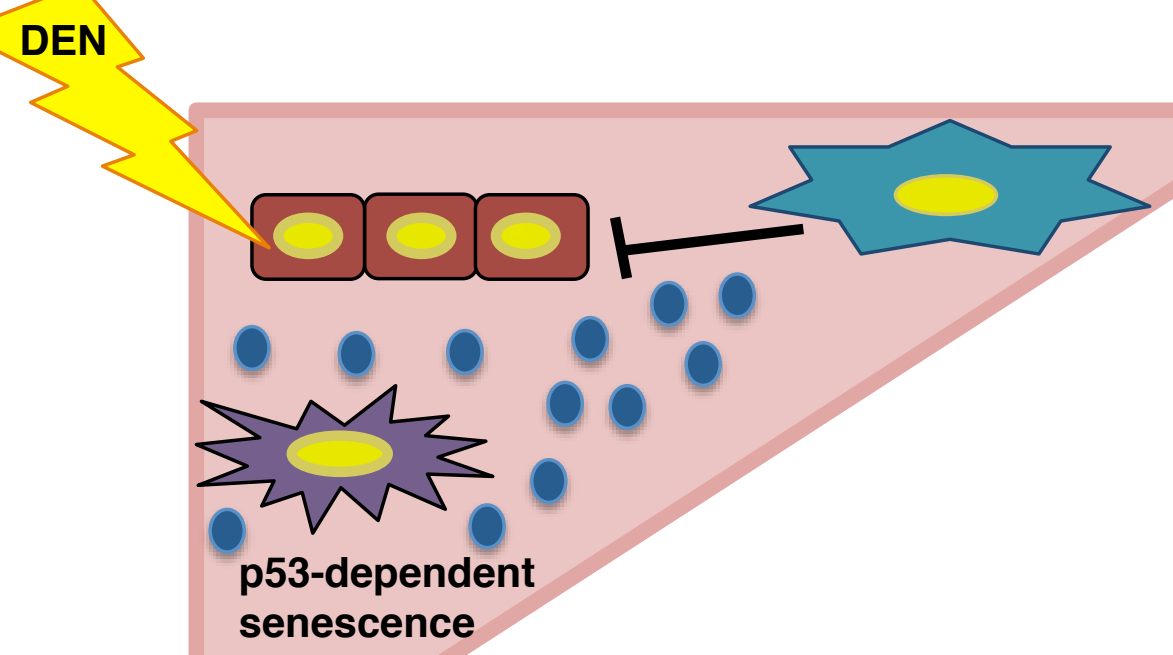

3. Senescent hepatic stellate cells increase $\mathrm{HCC}$ in obese liver
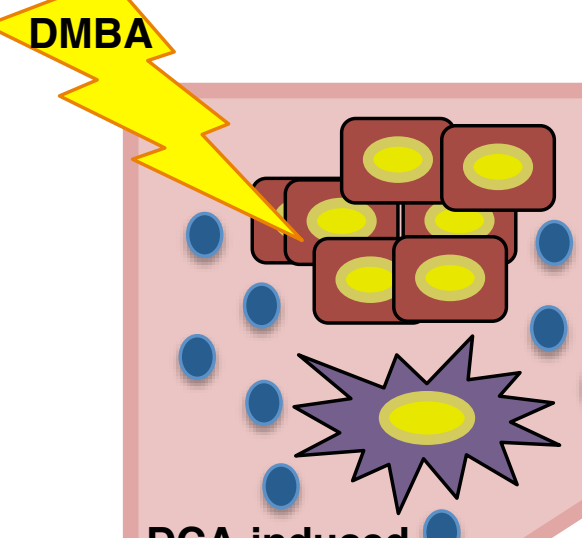

DCA-induced

senescence
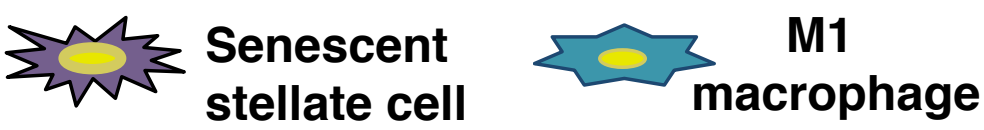

initiated hepatocyte 
Figure 3

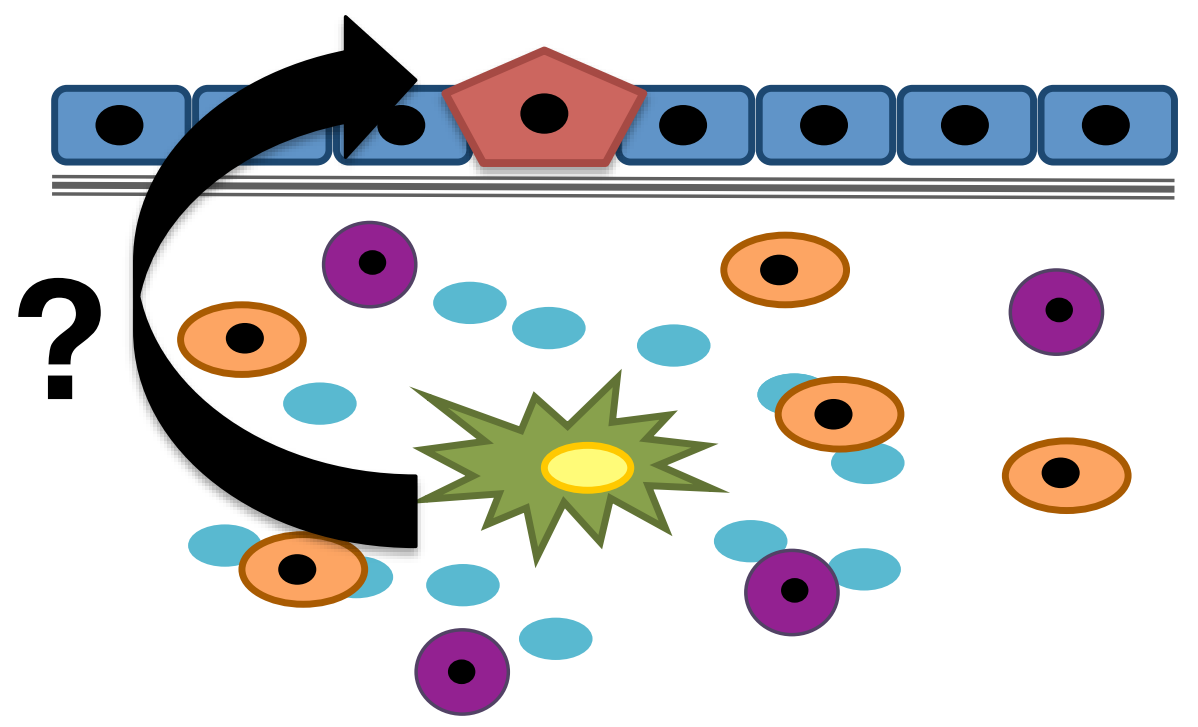

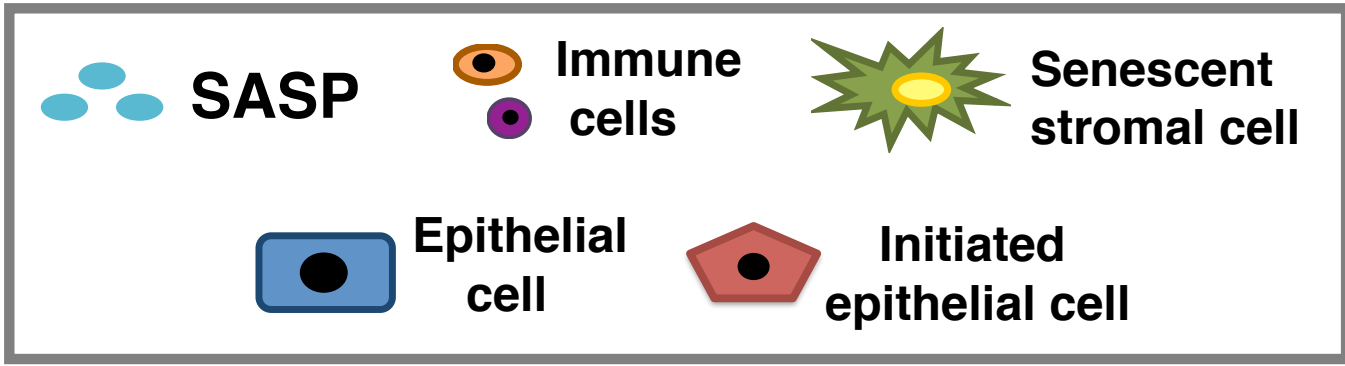

